\title{
Paper Suitable Spreading Sequences for Asynchronous MC-CDMA Systems
}

\author{
Mouad Addad and Ali Djebbari \\ Faculty of Electrical Engineering, Djillali Liabes University of Sidi Bel Abbes, Sidi Bel Abbès, Algeria
}

https://doi.org/10.26636/jtit.2018.118217

\begin{abstract}
In order to meet the demand of high data rate transmission with good quality maintained, the multi-carrier code division multiple access (MC-CDMA) technology is considered for the next generation wireless communication systems. However, their high crest factor $(\mathrm{CF})$ is one of the major drawbacks of multi-carrier transmission systems. Thus, $\mathrm{CF}$ reduction is one of the most important research areas in MC-CDMA systems. In addition, asynchronous MC-CDMA suffers from the effect of multiple access interference (MAI), caused by all users active in the system. Degradation of the system's bit error rate (BER) caused by MAI must be taken into consideration as well. The aim of this paper is to provide a comparative study on the enhancement of performance of an MC-CDMA system. The spreading sequences used in CDMA play an important role in $\mathrm{CF}$ and interference reduction. Hence, spreading sequences should be selected to simultaneously ensure low $C F$ and low BER values. Therefore, the effect that correlation properties of sequences exert on $\mathrm{CF}$ values is investigated in this study. Furthermore, a numerical BER evaluation, as a function of the signal-to-noise ratio (SNR) and the number of users, is provided. The results obtained indicate that a trade-off between the two criteria is necessary to ensure good performance. It was concluded that zero correlation zone (ZCZ) sequences are the most suitable spreading sequences as far as the satisfaction of the above criteria is concerned.
\end{abstract}

Keywords-BER, crest factor, multiple access interference, $M C$ CDMA, peak-to-average power ratio, zero-correlation zone.

\section{Introduction}

Multi-carrier (MC) is the predominant transmission technique in today's communication systems. In particular, the multi-carrier code division multiple access (MC-CDMA) transmission scheme combines orthogonal frequency division multiplexing (OFDM) and code division multiple access (CDMA). The main advantages of the combined system are: variable data rate, high spectral efficiency and robustness against frequency selective fading [1]-[7]. However, one major drawback of this technology is the large peak-to-average power ratio (PAPR). High PAPR reduces power efficiency and causes implementation-related issues [3]. Therefore, achieving very low PAPR values is of major importance. The unique structure of CDMA can be utilized by considering the signal design aspect. PAPR can be minimized through finding a suitable family of sequences [3], [4]. MC-CDMA encounters another problem, i.e. the multiple access interference (MAI). This type of interference occurs when system users are transmitting asynchronously, i.e. there is a timing misalignment among users, such as the uplink channel of cellular mobile systems. To mitigate MAI, several techniques were proposed recently [1], [5], [6]. However, these techniques only add to the complexity of MC-CDMA systems. In contrast, MAI interference could be eliminated through the design of a set of sequences with impulsive auto-correlation function (ACF) and zero cross-correlation functions (CCF) [6], [8]. Unfortunately, such ideal sequences are impossible to construct [8]. Instead, various sequences could be used: Walsh-Hadamard (WH), orthogonal Gold, orthogonal Golay complementary (OGC), and zero-correlation zone (ZCZ). Each sequence set has specific ACF and CCF properties. ZCZ sequences have been proposed and studied in [9]-[13].

The objective of this paper is to investigate the performance of ZCZ sequences based on two criteria: PAPR and bit error rate (BER) in an MC-CDMA system. In this study, we consider two MAI effects, i.e. inter-symbol interference (ISI) and inter-channel interference (ICI). Furthermore, we evaluate the performance of ZCZ sequences against conventional sequences. The sequence set with both a low crest factor and low BER performance is defined as optimal.

The remaining content of the paper is organized as follows. In Section 2 we give preliminaries to correlation functions, sequences generation, and the concept of an MC-CDMA system. In Section 3, the performance of ZCZ sequences in terms of both PAPR and BER is evaluated. Finally, concluding remarks on the optimal sequences are given in Section 4.

\section{Preliminaries}

First, the correction function are defined. A sequence of length $N$ is denoted by $\mathbf{a}_{N}=\left[a_{0}, a_{1}, \ldots, a_{n}, \ldots, a_{N-1}\right]$. A set of $M$ sequences $\left\{\mathbf{a}^{1}, \mathbf{a}^{2}, \ldots, \mathbf{a}^{M}\right\}$ is denoted by $\left\{\mathbf{a}^{m}\right\}_{m=1}^{M}$. The discrete periodic $R_{\mathbf{a}^{x}} \mathbf{a}^{y}(\tau)$ and aperiodic $\theta_{\mathbf{a}^{x}} \mathbf{a}^{y}(\tau)$ crosscorrelation functions of a pair of sequences $\mathbf{a}^{x}$ and $\mathbf{a}^{y}$ are defined as [14]:

$$
\begin{gathered}
R_{\mathbf{a}^{x} \mathbf{a}} y=\sum_{n=0}^{N-1} a_{n}^{x}\left[a_{(n+\tau) \bmod N}^{y}\right]^{*}, \\
\theta_{\mathbf{a}^{x_{\mathbf{a}}} y}(\tau)=\sum_{n=0}^{N-1-\tau} a_{n}^{x}\left[a_{n+\tau}^{y}\right]^{*},
\end{gathered}
$$


where $\tau$ is the sequence shift variable and $\left[a_{n}\right]^{*}$ denotes the complex conjugate of sequence element $a_{n}$. The notation (.) $\bmod N$ denotes a modulo $N$ operation. When $x=y$, CCF becomes ACF and will be denoted simply by $R_{\mathbf{a}^{x}}(\tau)$ and $\theta_{\mathbf{a}^{x}}(\tau)$.

Next, the ZCZ sequences are defined using binary construction methods. A set of binary sequences $\left\{\mathbf{a}^{m}\right\}_{m=1}^{M}$ that satisfy the following conditions is a $\mathrm{ZCZ}$ sequence set denoted by $Z C Z\left(N, M, Z_{0}\right)$, where $Z_{0}$ is the zeros zone length [8].

$$
R_{\mathbf{a}^{x}, \mathbf{a}^{y}}(\tau)= \begin{cases}N & \tau=0, x=y \\ 0 & \tau=0, x \neq y \\ 0 & 0<|\tau| \leq Z_{0}\end{cases}
$$

where $\tau=0$ means that CCF is computed at the in-phase. Among the flexible construction methods considered in this study are those proposed in [11]-[13].

WH sequence set is obtained directly from the Hadamard matrix. Any pair of sequences verifies the following property [15]:

$$
R_{\mathbf{a}^{x} \mathbf{a}}(\tau)= \begin{cases}N & \tau=0 x=y \\ 0 & \tau=0, x \neq y\end{cases}
$$

The orthogonal Gold sequences are generated from their corresponding Gold sequences [14] by appending +1 at the subset end [16].

OGC can be generated using the following recursive method [15]:

$$
\mathbf{a}_{2 N}=\left[\begin{array}{cc}
\mathbf{a}_{N} & \overline{\mathbf{a}}_{N} \\
\mathbf{a}_{N} & -\overline{\mathbf{a}}_{N}
\end{array}\right]
$$

where $\mathbf{a}_{N}$ is a square matrix of order $N$ with complementary rows. Two binary sequences $\left(\mathbf{a}^{x}, \mathbf{a}^{y}\right)$ of length $N$ are complementary if the sum of their aperiodic ACFs satisfies [14]:

$$
\theta_{\mathbf{a}^{x}}(\tau)+\theta_{\mathbf{a}^{y}}(\tau)= \begin{cases}2 N & \tau=0 \\ 0 & \tau \neq 0\end{cases}
$$

The $\overline{\mathbf{a}}_{N}$ means that the right half columns of $\mathbf{a}_{N}$ are reversed.

In the MC-CDMA scheme of $K$ users, the same information symbol $b_{k}(m)$ is spread over $N$ carriers, each multiplied by a different element of the spreading sequence $\mathbf{c}_{k}=\left\{c_{k, n}\right\}_{n=1}^{N}$ assigned to user $k$. After spreading, the user bit is modulated onto successive subcarriers such that one information symbol is spread over several subcarriers. Binary phase-shift keying (BPSK) modulation is used. The MC-CDMA transmitter for user $k$ is shown in Fig. 1.

The transmitted signal for user $k$ is [16], [17]:

$$
s_{k}(t)=\sqrt{\frac{2 P}{N}} \sum_{m=-\infty}^{\infty} b_{k}(m) u_{T_{b}}\left(t-m T_{b}\right) \sum_{n=1}^{N} c_{k, n} \mathrm{e}^{\mathrm{j}\left(w_{n} t+\theta_{k}\right)}
$$

where $P$ is the power of data bits, $u_{T_{b}}(t)$ is the rectangular pulse defined in $\left[0, T_{b}\right]$ with $T_{b}$ denoting the bit duration, $w_{n}=\frac{2 \pi n}{T_{b}}$ is the $n$-th subcarrier angular frequency, and the random phase $\theta_{k}$ is uniformly distributed over $[0,2 \pi]$. The

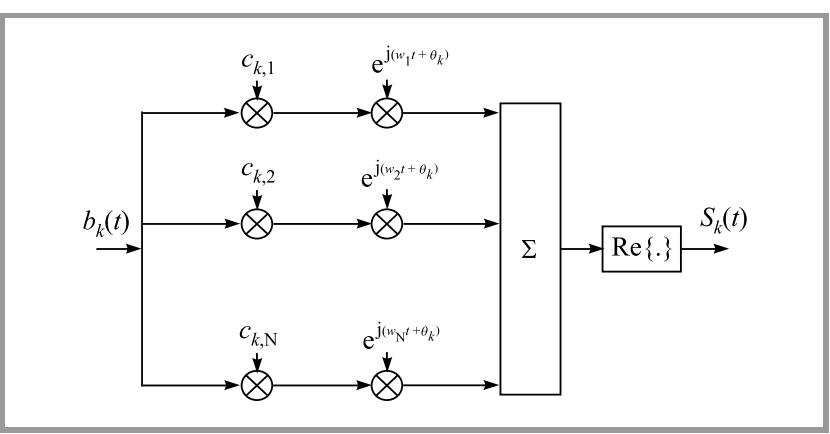

Fig. 1. MC-CDMA Transmitter for the $k$-th user.

neighboring subcarriers are orthogonal and separated by $\Delta w=\frac{2 \pi}{T_{b}}$.

An asynchronous MC-CDMA system in the additive white Gaussian noise (AWGN) channel is considered. We restrict our analysis to line of sight conditions where the multipath replicas have insignificant power compared to the line of sight signal [18]. The received signal can be written as [16], [17]:

$$
\begin{aligned}
r(t)=\sqrt{\frac{2 P}{N}} \sum_{k=1}^{K} \sum_{m=-\infty}^{\infty} b_{k}(m) u_{T_{b}}\left(t-m T_{b}-\tau_{k}\right) \\
\times \sum_{n=1}^{N} c_{k, n} \mathrm{e}^{\mathrm{j}\left(w_{n} t+\varphi_{k, n}\right)}+n(t),
\end{aligned}
$$

where $\tau_{k}$ is the random time delay of user $k$ uniformly distributed over bit duration $\left[0, T_{b}\right], \varphi_{k, n}=\theta_{k}-w_{n} \tau_{k}$, and $n(t)$ is is a zero-mean bandpass white noise with equivalent low pass signal that has a power spectral density $N_{0}$. The MC-CDMA receiver for the $i$-th user is shown in Fig. 2.

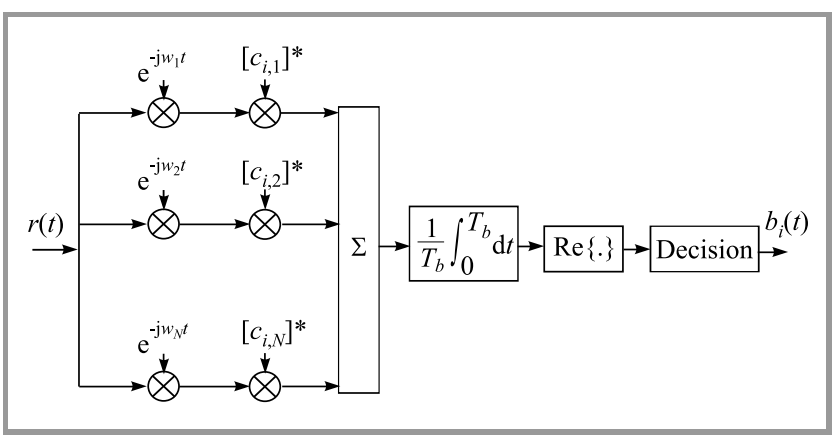

Fig. 2. MC-CDMA receiver for the $i$-th user.

We consider a coherent correlation receiver synchronized to the desired user $i\left(\tau_{i}=0\right)$. Time delays are quantized to integer multiple of the code element (chip) duration $T_{c}=\frac{T_{b}}{N}$. The decision variable of the 0 th data bit of user $i$ is given by [16]-[17]:

$$
\begin{array}{r}
Z_{i, 0}=\operatorname{Re}\left\{\frac{1}{T_{b}} \int_{0}^{T_{b}} \sum_{n^{\prime}=1}^{N} r(t)\left[c_{i, n^{\prime}}\right]^{*} \mathrm{e}^{-\mathrm{j} w_{n^{\prime}} t} \mathrm{~d} t\right\} \\
=D_{i}+n_{i}+M A I_{i},
\end{array}
$$

where $D_{i}, n_{i}, M A I_{i}$ are the desired signal, the AWGN, and the multiple access interference terms, respectively. With- 
out any loss of generality, we divide both sides of Eq. (9) by $\sqrt{\frac{2 P}{N}}$.

The desired symbol $b_{i, 0}$ is influenced by the corresponding $b_{k, 0}$ and previous symbols $b_{k,-1}$ of each interferer $k$, thus the interference between users at the access point or multiple access interference $M A I_{i}$ term is given by [17]:

$$
\begin{aligned}
& M A I_{i}=\operatorname{Re}\left\{\frac { 1 } { T _ { b } } \sum _ { n ^ { \prime } = 1 } ^ { N } [ c _ { i , n ^ { \prime } } ] ^ { * } \sum _ { \substack { k = 1 \\
k \neq i } } ^ { K } \sum _ { n = 1 } ^ { N } c _ { k , n } \left[b_{k,-1}\right.\right. \\
& \left.\left.\times \int_{0}^{\tau_{k}} \mathrm{e}^{\mathrm{j}\left(\left[w_{n}-w_{n^{\prime}}\right] t+\varphi_{k, n}\right)} \mathrm{d} t+b_{k, 0} \int_{\tau_{k}}^{T_{b}} \mathrm{e}^{\mathrm{j}\left(\left[w_{n}-w_{n^{\prime}}\right] t+\varphi_{k, n}\right)} \mathrm{d} t\right]\right\} .
\end{aligned}
$$

Or, in a compact form:

$$
M A I_{i}=\sum_{\substack{k=1 \\ k \neq i}}^{K} M A I_{i, k}=\sum_{\substack{k=1 \\ k \neq i}}^{K} I S I_{i, k}+I C I_{i, k}
$$

We can distinguish two cases: $n=n^{\prime}$ and $n \neq n^{\prime}$. In the first case, interference from the same subcarriers of user $k$ is called inter-symbol interference (ISI). If $n \neq n^{\prime}$, interference from other subcarriers of user $k$ is called inter-channel interference (ICI).

$$
\begin{aligned}
I S I_{i, k}=\operatorname{Re} & \left\{\frac { 1 } { T _ { b } } \sum _ { n = 1 } ^ { N } [ c _ { i , n } ] ^ { * } c _ { k , n } \left[b_{k,-1} \tau_{k}\right.\right. \\
& \left.\left.+b_{k, 0}\left(T_{b}-\tau_{k}\right)\right] \mathrm{e}^{\mathrm{j} \varphi_{k, n}}\right\}, \\
I C I_{i, k}=\operatorname{Re} & \left\{\frac{2}{T_{b}} \sum_{n^{\prime}=1}^{N} \sum_{\substack{n=1 \\
n \neq n^{\prime}}}^{N}\left[c_{i, n^{\prime}}\right]^{*} c_{k, n} \frac{b_{k,-1}-b_{k, 0}}{w_{n}-w_{n^{\prime}}}\right. \\
& \left.\times\left[e^{\mathrm{j}\left(\frac{\left[w_{n}-w_{n^{\prime}}\right] \tau_{k}}{2}+\varphi_{k, n}\right)} \sin \frac{\left[w_{n}-w_{n^{\prime}}\right] \tau_{k}}{2}\right]\right\} .
\end{aligned}
$$

It is worth noting that for a synchronous transmission $\left(\tau_{k}=\right.$ $0, \forall k)$, ICI does not exist and the ISI reduces to:

$$
I S I_{i, k}=\operatorname{Re}\left\{b_{k, 0} \mathrm{e}^{\mathrm{j} \theta_{k}} \sum_{n=1}^{N}\left[c_{i, n}\right]^{*} c_{k, n}\right\} .
$$

Thus, we can conclude that the offset (time delays) between users generates ICI and degrades BER performance, as shown in [17]. In a synchronous case, ISI can be eliminated using a set of orthogonal sequences.

Since we considered an AWGN channel, the decision variable can be regarded as a Gaussian random variable [17]. The BER of user $i$, conditioned on $\left\{\tau_{k}\right\},\left\{\theta_{k}\right\},\left\{b_{k,-1}\right\}$, and $\left\{b_{k, 0}\right\}$, is [17]:

$$
P_{e, i / \tau_{k}, \theta_{k}, b_{k,-1}, b_{k, 0}(k \neq i)}=\frac{1}{2} \operatorname{erfc}\left[\sqrt{\frac{E_{b}}{N_{0}}}\left(1+\frac{M A I_{i}}{N}\right)\right],
$$

where $\frac{E_{b}}{N_{0}}$ is defined as SNR. To obtain the unconditional BER for user $i$, we average $P_{e, i / \tau_{k}, \theta_{k}, b_{k,-1}, b_{k, 0}(k \neq i)}$ over all variables via Monte Carlo integration. A more appropriate performance measure is the averaged BER over all users [17]:

$$
P_{e}=\frac{1}{K} \sum_{i=1}^{K} P_{e, i}
$$

Note that the results obtained for an asynchronous transmission using the Gaussian model do agree with system simulations for a relatively large number of users [19].

Another important criterion for the selection of spreading sequences for MC-CDMA is PAPR. The PAPR parameter of a signal is defined as the ratio of peak to average signal power. In this study, we consider crest factor (CF) as a measure of signal envelope compactness, its relation to PAPR is [16]:

$$
C F=\sqrt{P A P R} .
$$

The CF of an MC-CDMA signal must satisfy the following inequality [16]:

$$
\mathrm{CF} \leq \frac{\max |S(f)|}{\sqrt{\frac{E}{2}}}
$$

where $S(f)$ is the Fourier transform of the spreading sequences and $E$ is the energy of the same sequence. The Fourier transform of the spreading sequence can be calculated by applying the well-known auto-correlation theorem, given by [16]:

$$
|S(f)|^{2}=\sum_{\tau=-(N-1)}^{N-1} \theta_{\mathbf{a}^{x}}(\tau) \mathrm{e}^{-\mathrm{j} 2 \pi \tau f} .
$$

\section{Numerical Analysis}

In this section, the conventional sequences in CDMA systems and $\mathrm{ZCZ}$ sequences are analyzed in terms of $\mathrm{CF}$ and BER. First, we evaluate the $\mathrm{CF}$ values of various sequences and then simulate their performance in an asynchronous MC-CDMA environment.

\subsection{CF Analysis}

The evaluation procedure of $\mathrm{CF}$ values is performed as follows. First, sequence sets each of lengths $N=8,16,32$, 64 , and 128 , were generated. Second, the CF is computed for each sequence in the set and all values are averaged. Figure 3 illustrates $\mathrm{CF}$ performance of various sequences. Note that as the sequence's length increases, CF increases as well, except for OGC for $N \geq 32$ and ZCZ in [12] for $N \geq 64$ where it remains constant. In addition to their low $\mathrm{CF}$ levels, OGC and ZCZ in [12] also have a steady CF, which constitutes a major advantage in MC-CDMA systems. Note also that only OGC and ZCZ sequences remain under $\mathrm{CF}$ value of 3 for all lengths. The second-best sequences are those in [11] and the well known orthogonal Gold. The CF level for these codes is relatively low and slowly increases with the increase of sequence's length $N$. WH sequences have, by far, the highest values of CF. 


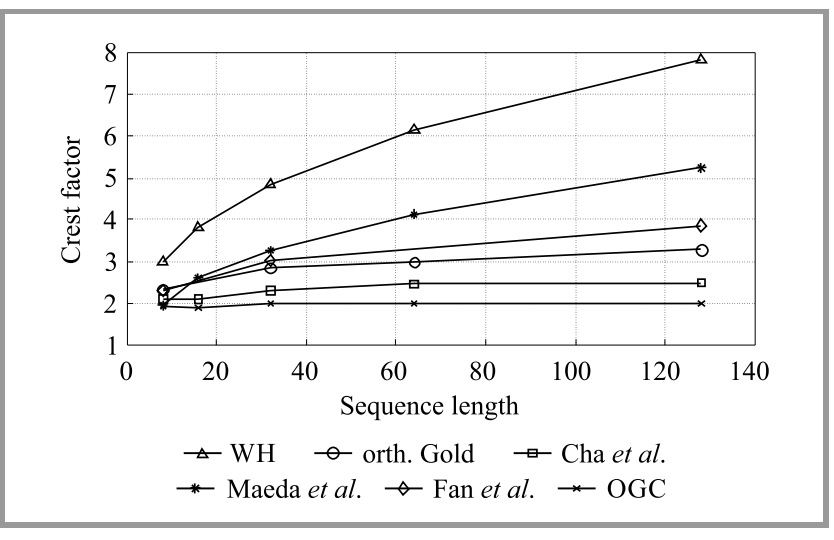

Fig. 3. Average $\mathrm{CF}$ of sequences for various lengths.

\subsection{BER Analysis}

This section focuses on interference related issues in asynchronous MC CDMA systems. The performance of an MCCDMA system using various spreading codes in the presence of MAI in an AWGN channel is simulated using Matlab. We have considered an asynchronous MC-CDMA system, where a number of mobile users transmit their signal to a common receiver such as the uplink cellular system. First, the effect of the sequence length on BER performance of 4 users was analyzed and plotted in Fig. 4. For simulation, orthogonal Gold sequences were used with lengths 7, 31, and 63. As it can be seen in Fig. 4, the BER performance improves with the increase of the sequence length. To obtain a BER of $10^{-4}$, MC-CDMA using sequences of length $N=63$ requires $2 \mathrm{~dB}$ less SNR than sequence of length $N=31$.

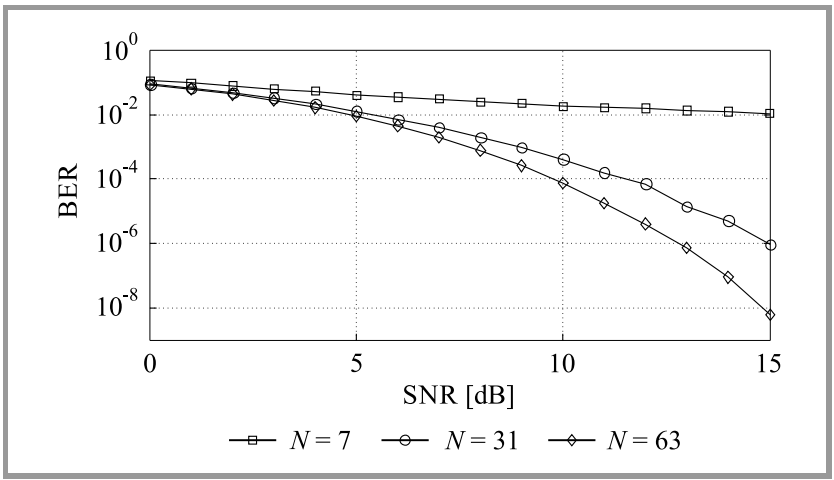

Fig. 4. BER versus SNR at different lengths of orthogonal Gold spreading sequence.

The effect of the number of active users on BER performance is analyzed next. Figure 5 shows BER performance of MC-CDMA versus the number of users at different values of $\mathrm{SNR}=5,10$, and 15. For simulation, orthogonal Gold sequences were used $(N=31)$. As anticipated, a high number of active users causes degradation in BER performance. This is because admitting more users to the system results in higher level of interference (MAI).

We conclude this section with a comparative study of various spreading sequences. In this simulation, the number

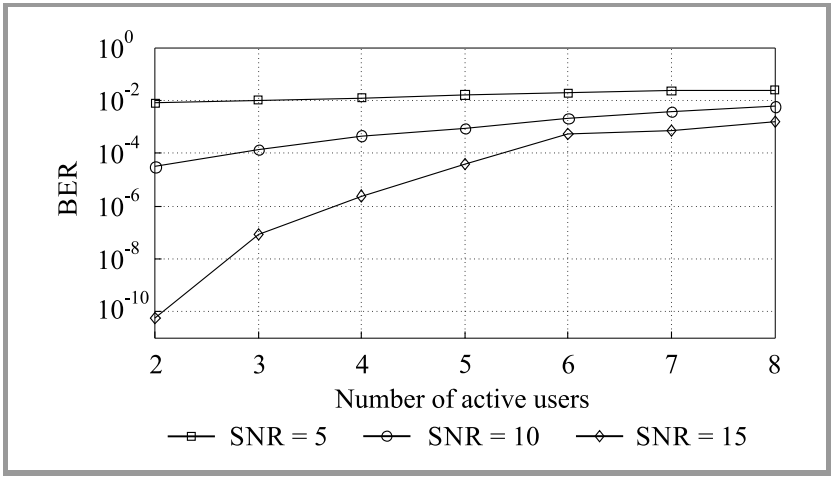

Fig. 5. BER versus number of active users for orthogonal Gold sequence at different SNR values.

of users is $K=8$, the sequence length is $N=32$ for WH, ZCZ and OGC sequences and $N=31$ for orthogonal Gold sequences, and the bit rate is taken as $100 \mathrm{Mbps}$. For $\mathrm{SNR} \geq 20 \mathrm{~dB}, \mathrm{BER}$ remains constant and is equal to $5 \cdot 10^{-3}$ for $\mathrm{WH}$ and OGC sequences, $2 \cdot 10^{-3}$ for $\mathrm{ZCZ}$ sequences and $7.5 \cdot 10^{-4}$ for orthogonal Gold sequences. From the results obtained, it is observable that Gold outperforms WH and OGC sequences by a factor of 6.6 and $\mathrm{ZCZ}$ by a factor of 2.6.

\subsection{Discussion}

According to the BER criterion, the best results are obtained when orthogonal Gold sequences are used for

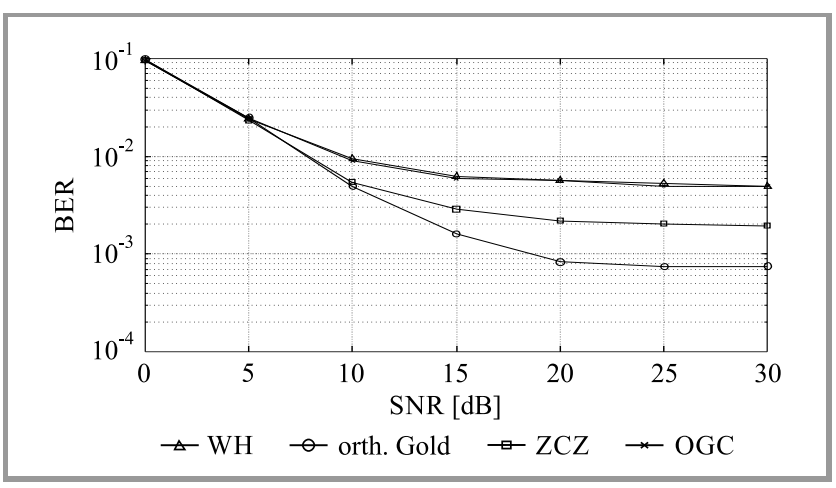

Fig. 6. BER versus SNR curves of various spreading sequences.

spreading (Fig. 6). In addition, BER performance of the system also improves with the increase of the spreading sequence length (Fig. 4). However, MC-CDMA systems using orthogonal Gold sequences suffer from considerably

Table 1

System parameters

\begin{tabular}{|c|c|c|}
\hline Sequences & CF & BER \\
\hline \hline ZCZ & Low & Low \\
\hline WH & Highest & Highest \\
\hline $\begin{array}{c}\text { Orthogonal } \\
\text { Gold }\end{array}$ & High & Lowest \\
\hline OGC & Lowest & High \\
\hline
\end{tabular}


high values of $\mathrm{CF}$, which leads to signal distortion (Fig. 3). Moreover, increasing the sequence length would lead to even higher values of $\mathrm{CF}$ in the case of orthogonal Gold sequences (Fig. 3). Thus, a trade-off must be established between low BER and low CF. From the simulation results, it can be observed that ZCZ sequences have both low BER and low CF (see Table 1). Therefore, we conclude that $\mathrm{ZCZ}$ sequences are the suitable candidate for asynchronous MC-CDMA systems.

\section{Conclusion}

This work was carried out to enhance the performance of an MC-CDMA system through the proper choice of the spreading sequences. The performance of CDMA-based wireless systems is mainly governed by the characteristics of the spreading sequences i.e. their length and correlation properties. In this paper, $\mathrm{CF}$ performance of $\mathrm{ZCZ}$, WH, orthogonal Gold, and OGC sequences was analyzed and compared. In addition, BER performance of an asynchronous MC-CDMA system in the AWGN channel was evaluated as well. With the use of an efficient spreading sequence, the system enjoys low CF and BER levels. Based on the results obtained for different sequences, it is observed that BER performance of the system degrades with an increase of the number of users and improves with an increase the spreading sequence length. The system with ZCZ sequences shows good performance in terms of both $\mathrm{CF}$ and BER. We conclude that ZCZ sequences have the best trade-off possible and therefore are the suitable candidate for asynchronous MC-CDMA systems.

\section{References}

[1] K. S. Vishvaksenan and P. T. Vasanth Raj, "Coded downlink multiuser MC-CDMA system using transmitter pre-processing: Performance results", IEEE Access, vol. 4, pp. 4534-4542 (doi: 10.1109/ACCESS.2016.2596774).

[2] J. O. M. Amok and N. M. Saad, "Error rate performance analysis of multiantenna MC DS-CDMA system over $\mu-\mu$ frequency selective fading channels with arbitrary parameters", Int. J. of Electron. Commun., vol. 70, no. 5, pp. 517-529, 2015 (doi: 10.1016/j.aeue.2015.11.007).

[3] W.-J. Huang, W.-W. Hu, C.-P. Li, and J.-C. Chen, "Novel metricbased PAPR reduction schemes for MC-CDMA systems", IEEE Transact. on Vehic. Technol., vol. 64, no. 9, pp. 3982-3989, 2015 (doi: 10.1109/TVT.2014.2361353).

[4] H.-H. Chang, S.-C. Lin, and C.-D. Lee, "A CDMA scheme based on perfect Gaussian integer sequences", Int. J. of Electron. Commun., vol. 75, pp. 70-81, 2017 (doi: 10.1016/j.aeue.2017.03.008).

[5] R. AliHemmati, P. Azmi, and F. Marvasti, "Joint multi-user interference and clipping noise cancellation in uplink MC-CDMA system", Int. J. of Electron. Commun., vol. 64, no. 5, pp. 425-432, 2010 (doi: 10.1016/j.aeue.2009.02.006)
[6] D. Judson and A. Albert, "Performance of multicarrier complementary-coded CDMA under frequency-selective Nakagami- $m$ fading channels", EURASIP J. of Wireless Commun. and Network., vol. 67, pp. 1-9, 2016 (doi: 10.1186/s13638-016-0563-y).

[7] A. B. Djebbar, A. Djebbari, M. Bouziani, and J. M. Rouvaen, "Derivation of new expressions of bit error rate for MC-CDMA system in Nakagami fading channel", Int. J, of Electron. Commun., vol. 57 , no. 6 , pp. 395-402, 2003 (doi: 10.1078/1434-8411-54100191).

[8] P. Fan, "Spreading sequence design and theoretical limits for quasisynchronous CDMA", EURASIP J. of Wireless Commun. and Network., vol. 2004, no. 1, pp. 19-31, 2014 (doi: 10.1155/S1687147204405015).

[9] M. Addad and A. Djebbari, "A ternary zero correlation zone sequence sets construction procedure", Turkish J. of Electr. Engin. Comp. Sciences, vol. 25, no. 3, 2016 (doi: 10.3906/elk-1603-115).

[10] Z. Zhang, L. Ge, F. Zeng, and G. Xuan, "Zero correlation zone sequence set with inter-group orthogonal and inter-subgroup complementary properties", Adv. in Math. of Commun., vol. 9, no. 1, pp. 9-21, 2015 (doi: 10.3934/amc.2015.9.9).

[11] P. Fan, N. Suehiro, N. Kuroyanagi, and X. Deng, "Class of binary sequences with zero correlation zone”, Electron. Let., vol. 35, no. 10, pp. 777-779, 1999 (doi: 10.1049/el:19990567).

[12] J. Cha, S. Kameda, M. Yokoyama, H. Nakase, K. Masu, and K. Tsubouchi, "New binary sequences with zero-correlation duration for approximately synchronised CDMA", Electron. Let., vol. 36, no. 11, pp. 991-993, 2000 (doi: 10.1049/el:20000703).

[13] T. Maeda, S. Kanemoto, and T. Hayashi, "A novel class of binary zero-correlation zone sequence set", in Proc. 2010 IEEE Region 10 Conf. TENCON 2010, Fukuoka, Japan, 2010, pp. 708-711 (doi: 10.1109/TENCON.2010.5686619).

[14] P. Fan and M. Darnell, Sequence Design for Communications Applications, 1st ed. Baldock, UK: Research Studies Press, Electronic \& Electrical Engineering Research Studies. Communications Systems, Techniques, and Applications Series, 1, 1996 (ISBN: 978-0863802010).

[15] H. Ochiai and H. Imai, "OFDM CDMA with peak power reduction based on the spreading sequences", in Proc. 1998 IEEE int. Conf on Commun. (ICC'98), Atlanta, GA, USA, 1998, pp. 1299-1303 (doi: 10.1109/ICC.1998.683035).

[16] B. M. Povovic, "Spreading sequences for multicarrier CDMA systems", IEEE Transact. on Commun., vol. 47, no. 6, pp. 918-926, 1999 (doi: 10.1109/26.771348).

[17] Q. Shi and M. Latva-aho, "Spreading sequences for asynchronous MC-CDMA revisited: Accurate bit error rate analysis", IEEE Transact. on Commun., vol. 51, no. 1, pp. 8-11, 2003 (doi: 10.1109/TCOMM.2002.807631).

[18] D. Boudreau et al., "Wide-band CDMA for the UMTS/IMT-2000 satellite component", IEEE Transact. on Vehic. Technol., vol. 51, no. 2, pp. 306-331, 2002 (doi: 10.1109/25.994808).

[19] Q. Shi and M. Latva-aho, "Performance analysis of MC-CDMA in Rayleigh fading channels with correlated envelopes and phases", IEE Proc. - Commun., vol. 150, no. 3, pp. 214-220 (doi: 10.1049/ip-com:20030177).

Mouad Addad, Ali Djebbari - for biographies, see this issue, p. 8. 